Citation: Dağdeviren, A., Kürüz, F., "Special Real and Dual Matrices with Hadamard Product". Journal of Engineering Technology and Applied Sciences 6 (2) 2021 : 127-134.

\title{
SPECIAL REAL AND DUAL MATRICES WITH HADAMARD PRODUCT
}

\author{
Ali Dă̆deviren ${ }^{\text {a* }}$ (D) Ferhat Kürüz ${ }^{\mathbf{b}}$ \\ $a^{*}$ Department of Weight and Balance, Turkish Aviation Academy, \\ 34149 Bakurköy, Istanbul, Turkey \\ adagdeviren@thy.com (*corresponding author) \\ ${ }^{b}$ Department of Computer Engineering, Faculty of Engineering and Architecture \\ Istanbul Gelisim University, 34310 Istanbul, Turkey \\ fkuruz@gelisim.edu.tr
}

\begin{abstract}
In this paper, firstly we will present basic properties of Hadamard matrix product and Dual matrices to built necessary background. Then we will define special real and dual matrices under this matrix product. Finally, some theorems regarding this matrix product will be given.
\end{abstract}

Keywords: Hadamard product, dual numbers, dual matrices

\section{Introduction}

The Hadamard product (1.1) of matrices is carried out entry by entry, as in traditional matrix addition. It is also known as Schur product, entrywise product, or the element-wise product which has a significant place in matrix theory and in statistical analysis [1].

In the literature, there are more than 400 scientific papers using Hadamard product about positive semidefinite matrix, upper bound for an arbitrary determinant with the help of Hadamard matrix product written by Jacques Hadamard. Issai Schur does not seem to know of any previous work on the product (1.1), which shows that as long as A and B are positive semi-definite, their product hadamard is also positive [2-4]. Hence, some mathematicians used the name Hadamard and the others used Schur product. On the other hand, there are many notation for using this product. Some of them are $\times, \odot, *, \circ$. In this paper we will use the name "Hadamard" and notation "o" for this product $[5,6]$. 
Dual numbers $(\mathbb{D})$ are an extension of real numbers like complex numbers. This number system had been defined by Clifford for geometrical and mechanical applications. Moreover, dual vector algebra is widely used in kinematics, mechanism and physics [7-9].

In this paper, we firstly give basic concepts and properties of Hadamard product besides dual numbers and their matrices in order to establish requiring background. Then, in section two we will define special real matrices such as symmetric matrices and orthogonal matrices under the Hadamard product. Finally, in last section we define the Hadamard product of dual matrices and some special matrices.

\subsection{Hadamard product and its properties}

Definition 1.1 Let $A=\left[a_{i j}\right]$ and $B=\left[b_{i j}\right]$ be $m \times n$ real matrices, then the Hadamard product of these matrices is defined as

$A \circ B=\left[a_{i j} b_{i j}\right]_{m \times n}$.

As it can be seen from the definition, in order to multiply to matrices with Hadamard product matrices must be in the same size. Similarly to matrix addition, Hadamard product is commutative [4].

Example 1.2 If $A=\left[\begin{array}{ccc}1 & 0 & 3 \sqrt{7} \\ 5 & -\sqrt{2} & 2\end{array}\right]$ and $B=\left[\begin{array}{ccc}-3 & 4 & \sqrt{7} \\ -2 & 8 & -1\end{array}\right]$ then the hadamard product of these matrices is $A \circ B=\left[\begin{array}{ccc}-3 & 0 & 21 \\ -10 & -8 \sqrt{2} & -2\end{array}\right]$.

It is clear that the set of $\mathbb{R}_{n}^{m}$ is a group with hadamard product, also it is easy to see that the triple $\left(\mathbb{R}_{n}^{m},+, \circ\right)$ is a commutative ring with identity. In addition, the set of $\mathbb{R}_{n}^{m}$ is an algebra over real numbers.

The identity matrix under the Hadamard product is called $H$ - identity matrix. All entries of $H$-identity matrix equal to 1 and $m \times n$ types $H$-identity matrix is denoted by $J_{n}^{m}$. If $A$ is a real matrix, $H$-identity matrix provides $A \circ J=J \circ A=A$. Null matrices in Hadamard multiplication retain their role. That is, $A \circ 0=0$.

Example 1.3 $J_{2}^{3}=\left[\begin{array}{lll}1 & 1 & 1 \\ 1 & 1 & 1\end{array}\right]$ is a $H$-identity matrix.

Hadamard product is associative and distributive over matrix addition:

- $A \circ B=B \circ A$

- $A \circ(B \circ C)=(A \circ B) \circ C$,

- $A \circ(B+C)=(A+B) \circ(A+C)$

where $A, B$ and $C \in \mathbb{R}_{n}^{m}$, [3, 6].

Definition 1.4 Let $A=\left[a_{i j}\right]$ be a real $m \times \mathrm{n}$ matrix. The inverse of $A$ with Hadamard product is denoted by $A^{-1}$ and defined with $A^{-1}=\left[\frac{1}{a_{i j}}\right]$. It is clear to see that any matrix $A$ has a Hadamard inverse if and only if every entry of $\mathrm{A}$ is not equal to zero. Furthermore, inverse matrix holds the equation $A \circ A^{-1}=\mathrm{J}$. 
Example 1.5 For $A=\left[\begin{array}{cc}1 & -8 \\ -1 & 1 / 5\end{array}\right]$ be a $2 \times 2$ matrix, then the $H$-inverse of $A$ is $A^{-1}=$ $\left[\begin{array}{cc}1 & -1 / 8 \\ -1 & 5\end{array}\right]$. There is no inverse of $B=\left[\begin{array}{ll}1 & 0 \\ 2 & 5\end{array}\right]$ under the Hadamard product, because it contains zero element.

Further informations about Hadamard product can be found in [4].

\subsection{Dual öatrices and their properties}

A dual number is generally denoted in the form $\hat{d}=a+\varepsilon a^{*}$, with a real unit 1 , and the dual unit $\varepsilon$, where $\varepsilon \neq 0, \varepsilon^{2}=\varepsilon^{3}=\cdots=0,0 \varepsilon=\varepsilon 0=0$ and $1 \varepsilon=\varepsilon 1=\varepsilon$. Moreover, every dual number can be illustrated by a pair of real numbers as $\left(a, a^{*}\right)$. The set of dual numbers $\mathbb{D}$ is defined as follows:

$\mathbb{D}=\left\{\hat{d}=a+\varepsilon b \mid a, b \in \mathbb{R}, \varepsilon^{2}=0, \varepsilon \neq 0\right\}$.

Equality, addition and multiplication of dual numbers $\hat{d}_{1}=a+\varepsilon a^{*}, \hat{d}_{2}=b+\varepsilon b^{*}$ are defined by $\hat{d}_{1}=\hat{d}_{2}$ iff $a=b$ and $a^{*}=b^{*}, \hat{d}_{1}+\hat{d}_{2}=(a+b)+\varepsilon\left(a^{*}+b^{*}\right)$ and $\hat{d}_{1} \cdot \hat{d}_{2}=$ $(a . b)+\varepsilon\left(a . b^{*}+a^{*} . b\right)$ respectively.

$(\mathbb{D},+,$.$) is a commutative ring with the unit element 1+\varepsilon 0$. Further information about dual numbers can be found in [7-9]. The set of all $m \times n$ real matrices is denoted by $\mathbb{R}_{n}^{m}$ and it is a vector space with matrix addition and scalar matrix multiplication. Furthermore, the set of $m \times n$ matrices with dual number entries is denoted by $\mathbb{D}_{n}^{m}$. A general dual matrix can be written as follows:

$\hat{A}=\left[\begin{array}{llll}a_{11}+\varepsilon a_{11}^{*} & a_{12}+\varepsilon a_{12}^{*} & \ldots & a_{1 n}+\varepsilon a_{1 n}^{*} \\ a_{21}+\varepsilon a_{21}^{*} & a_{22}+\varepsilon a_{22}^{*} & \ldots & a_{2 n}+\varepsilon a_{2 n}^{*} \\ \vdots & \vdots & & \vdots \\ a_{m 1}+\varepsilon a_{m 1}^{*} & a_{m 2}+\varepsilon a_{m 2}^{*} & \ldots & a_{m n}+\varepsilon a_{m n}^{*}\end{array}\right]$.

This dual matrix can be written dual combination of two real matrices as follows:

$$
\begin{aligned}
& \hat{A}=\left[\begin{array}{llll}
a_{11} & a_{12} & \ldots & a_{1 n} \\
a_{21} & a_{22} & \ldots & a_{2 n} \\
\vdots & \vdots & & \vdots \\
a_{m 1} & a_{m 2} & \ldots & a_{m n}
\end{array}\right]+\varepsilon\left[\begin{array}{llll}
a_{11}^{*} & a_{12}^{*} & \ldots & a_{1 n}^{*} \\
a_{21}^{*} & a_{22}^{*} & \ldots & a_{2 n}^{*} \\
\vdots & \vdots & & \vdots \\
a_{m 1}^{*} & a_{m 2}^{*} & \ldots & a_{m n}^{*}
\end{array}\right] \\
& \hat{A}=A+\varepsilon A^{*}
\end{aligned}
$$

where $A, A^{*} \in \mathbb{R}_{n}^{m}$. More properties about dual matrices can be found in $[7,10]$.

\section{Special real matrices with Hadamard product}

There are many kinds of special matrices with ordinary matrix multiplicationin the literature such as ortogonal, symmetric and periodic matrices. With this inspiration, we will define new special matrices with using hadamard matrix product. Under this matrix multiplication special 
matrices will called $H$-matrix. For example, we will call orthogonal matrix as $H-$ orthogonal matrix under Hadamard product.

Definition 2.1 An $m \times n$ matrix $A$ is called an $H$-singular matrix if the any entry of $A$ is zero. $A=\left[\begin{array}{ll}0 & 4 \\ 5 & 1\end{array}\right]$ is an $H$-singular matrix. Moreover, if there is no zero element in the matrix then it is called as regular matrix. $B=\left[\begin{array}{rr}2 & -3 \\ 1 & 6\end{array}\right]$ is a $H$-regular matrix.

Theorem 2.2 Let $A$ and $B$ be two $H$-regular matrices. Then following equality is hold

$$
(A \circ B)^{-1}=(B \circ A)^{-1}=A^{-1} \circ B^{-1}=B^{-1} \circ A^{-1}
$$

Proof. From the definition, we can write $(A \circ B) \circ(A \circ B)^{-1}=J$. Then using the associativity we have $A \circ\left(B \circ(A \circ B)^{-1}\right)=J$ and $B \circ(A \circ B)^{-1}=A^{-1}$. From this equality, we get the $(A \circ B)^{-1}=A^{-1} \circ B^{-1}$.

Proposition 2.3 For $A, B \in \mathbb{R}_{n}^{n}$, it is obvious that the trace of $A \circ B$ is equal to the trace of $B \circ$ $A$. That is, $\operatorname{tr}(A \circ B)=\operatorname{tr}(B \circ A)$.

Definition 2.4 For $A \in \mathbb{D}_{n}^{n}$, some special matrices can be defined as following:

i. If $A^{T}=A$, then $A$ is called $H$-symmetric matrix. Additionally, if $A$ is also a nonsingular, then $A^{T} \circ A^{-1}=J$.

ii. If $A^{T}=A^{-1}$, then $A$ is called $H$-antisymmetric matrix. Here, the diagonal entries of $A$ must be zero.

iii. If $A^{T} \circ A=J$, then $A$ is called $H$-orthogonal matrix.

Example 2.5 Examples of these special matrices can be given as following:

i. $\quad A=\left[\begin{array}{rrr}4 & -2 & 5 \\ -2 & 1 & -3 \\ 5 & -3 & 7\end{array}\right]$ is an $H-$ symmetric matrix.

ii. $B=\left[\begin{array}{rrr}1 & 2 & \frac{1}{5} \\ \frac{1}{2} & 1 & -3 \\ 5 & -\frac{1}{3} & 1\end{array}\right]$ is an $H-$ orthogonal matrix.

iii. $C=\left[\begin{array}{rrr}0 & -2 & 5 \\ 2 & 0 & 3 \\ -5 & -3 & 0\end{array}\right]$ is an $H$-antisymmetric matrix.

Theorem 2.6 Any matrix $A=\left[a_{i j}\right]$ is an $H$-orthogonal matrix if and only if $a_{i j} \cdot a_{j i}=1$.

Proof. With using definition $A^{T} \circ A=J$, we can write $\left[a_{i j}\right]^{T} \circ\left[a_{i j}\right]=[1]$. It is easy to see that $a_{i j} . a_{j i}=1$. Note that diagonal entries of $H$-orthogonal matrix is 1 .

Definition 2.7 Let $A$ be a $n \times n$ real matrix, then 
i. An $n \times n$ matrix $A$ is called $H$ - periodic matrix which satisfies the property $A^{k+1}=A$ with period $k$. If this equality holds for special case $k=1$, then the matrix $A$ is called $H$-idempotent. Note that these matrices' entries must be include just 1 . For instance $H$-identity matrix is an $H$-idempotent.

ii. An $n \times n$ matrix $A$ is called $H$-nilpotent matrix if $A^{2}=0$. There is no nilpotent matrix except zero matrix.

iii. An $n \times n$ matrix $A$ is called $H$-involute matrix if $A^{2}=J$. All elements of $H$-involut matrices are +1 or -1 .

Example 2.8 $A=\left[\begin{array}{rr}1 & -1 \\ -1 & -1 \\ -1 & 1\end{array}\right]$ is an $H$-involute matrix.

Definition 2.9 Let $A$ and $B$ be nonzero matrices. If $A \circ B=0, A$ and $B$ are called nulling each other matrices.

Example 2.10 Let $A=\left[\begin{array}{rrr}0 & 2 & -7 \\ 1 & 0 & 4\end{array}\right]$ and $B=\left[\begin{array}{ccc}3 & 0 & 0 \\ 0 & -1 & 0\end{array}\right]$ are two matrices. The Hadamard product of these matrices are $A \circ B=\left[\begin{array}{lll}0 & 0 & 0 \\ 0 & 0 & 0\end{array}\right]$. Therefore these matrices are nulling each other matrices.

\section{Hadamard product of dual matrices}

Let $\hat{A}=\left[a_{i j}+\varepsilon a_{i j}^{*}\right] \in \mathbb{D}_{n}^{m}$ and $\hat{B}=\left[b_{i j}+\varepsilon b_{i j}^{*}\right] \in \mathbb{D}_{n}^{m}$. These matrices can be written as the dual combination of two real matrices as $\hat{A}=A+\varepsilon A^{*}$ and $\hat{B}=B+\varepsilon B^{*}$ where $A, B, A^{*}$ and $B^{*}$ are real matrices. The Hadamard product of dual matrices $\hat{A}$ and $\hat{B}$ is defined as follows:

$$
\begin{aligned}
\hat{A} \circ \hat{B} & =\left[A_{i j}\right] \circ\left[B_{i j}\right]=\left[A_{i j} B_{i j}\right] \\
& =\left[\left(a_{i j}+\varepsilon a_{i j}^{*}\right) \cdot\left(b_{i j}+\varepsilon b_{i j}^{*}\right)\right] \\
& =\left[a_{i j} b_{i j}+\varepsilon\left(a_{i j} b_{i j}^{*}+a_{i j}^{*} b_{i j}\right)\right] \\
& =\left[a_{i j} b_{i j}\right]+\varepsilon\left[a_{i j} b_{i j}^{*}+a_{i j}^{*} b_{i j}\right] \\
& =A \circ B+\varepsilon\left(A \circ B^{*}+A^{*} \circ B\right) .
\end{aligned}
$$

Proposition 3.1 For every $\hat{A}, \hat{B}, \hat{C} \in \mathbb{D}_{n}^{m}$ following conditions are provided:

i. $\hat{A} \circ \hat{B}=\hat{B} \circ \hat{A}$,

ii. $\hat{A} \circ(\hat{B} \circ \hat{C})=(\hat{A} \circ \hat{B}) \circ \hat{C}$,

iii. $\hat{A} \circ(\hat{B}+\hat{C})=\hat{A} \circ \hat{B}+\hat{A} \circ \hat{C}$,

iv. For $k \in \mathbb{R}, k(\hat{A} \circ \hat{B})=(k \hat{A}) \circ \hat{B}=\hat{A} \circ(k \hat{B})$.

With the properties given here, it is seen that the set $\mathbb{D}_{n}^{m}$ is a ring with matrix addition and Hadamard product. From now on, we will use abbreviation $H$-product instead of Hadamard product.

Definition 3.2 A $m \times n \quad \mathbb{D}_{n}^{m}$-unit dual matrix according to $H$ - product, denoted by $\hat{J}_{n}^{m}$, is defined by 
$\hat{J}_{n}^{m}=\left[\begin{array}{cccc}1+\varepsilon 0 & 1+\varepsilon 0 & \ldots & 1+\varepsilon 0 \\ 1+\varepsilon 0 & 1+\varepsilon 0 & \ldots & 1+\varepsilon 0 \\ \vdots & \vdots & & \vdots \\ 1+\varepsilon 0 & 1+\varepsilon 0 & \ldots & 1+\varepsilon 0\end{array}\right]_{m \times n}$

Moreover, for every $\hat{A} \in \mathbb{D}_{n}^{m}$, we have $\hat{A} \circ \hat{J}_{n}^{m}=\hat{J}_{n}^{m} \circ \hat{A}=\hat{A}$.

Theorem 3.3 For $\hat{A}, \hat{B} \in \mathbb{D}_{n}^{n}$, the trace of $\hat{A} \circ \hat{B}$ is equal to the trace of $\hat{B} \circ \hat{A}$.

Proof.

$$
\begin{aligned}
\operatorname{tr}(\hat{A} \circ \hat{B}) & =\operatorname{tr}\left(A \circ B+\varepsilon\left(A \circ B^{*}+A^{*} \circ B\right)\right) \\
& =\operatorname{tr}(A \circ B)+\varepsilon \operatorname{tr}\left(A \circ B^{*}\right)+\varepsilon \operatorname{tr}\left(A^{*} \circ B\right) \\
& =\operatorname{tr}(B \circ A)+\varepsilon \operatorname{tr}\left(B^{*} \circ A\right)+\varepsilon \operatorname{tr}\left(B \circ A^{*}\right) \\
& =\operatorname{tr}(\hat{B} \circ \hat{A}) .
\end{aligned}
$$

Definition 3.4 Let $\hat{A} \in \mathbb{D}_{n}^{m}$. The conjugate of $\hat{A}$ is denoted $\overline{(\hat{A})}$ and defined $\overline{(\hat{A})}=A-\varepsilon A^{*}$.

Theorem 3.5 Let $\hat{A}=\left[A_{i j}+\varepsilon A_{i j}^{*}\right]=A+\varepsilon A^{*} \in \mathbb{D}_{n}^{m}$. If the real part of this dual matrix is $H$-invertiable, then $\hat{A}^{-1}=\frac{1}{A_{i j}^{2}} \overline{(\hat{A})}$ or $\hat{A}^{-1}=A^{-1}-\varepsilon A^{-2} \circ A_{i j}^{*}$.

Proof. From the conjugate of dual numbers we can write

$$
\hat{A}^{-1}=\left[\frac{1}{A_{i j}+\varepsilon A_{i j}^{*}}\right]=\left[\frac{A_{i j}-\varepsilon A_{i j}^{*}}{A_{i j}^{2}}\right]=\frac{1}{A_{i j}^{2}}\left[A_{i j}-\varepsilon A_{i j}^{*}\right]
$$

then we obtain

$$
\hat{A}^{-1}=\frac{1}{A_{i j}^{2}} \overline{(\hat{A})}=\hat{A}^{-1}=A^{-1}-\varepsilon \cdot A^{-2} \circ A^{*}
$$

Theorem 3.6 For $\hat{A}=A+\varepsilon A^{*}, \hat{B}=B+\varepsilon B^{*} \in \mathbb{D}_{n}^{m},(\hat{A} \circ \hat{B})^{T}=\hat{A}^{T} \circ \hat{B}^{T}$.

Proof. We know that $\hat{A} \circ \hat{B}=A \circ B+\varepsilon\left(A \circ B^{*}+A^{*} \circ B\right)$, the transpose of both sides of this equation is as follows:

$$
\begin{aligned}
(\hat{A} \circ \hat{B})^{T} & =\left(A \circ B+\varepsilon\left(A \circ B^{*}+A^{*} \circ B\right)\right)^{T} \\
& =(A \circ B)^{T}+\varepsilon\left(A \circ B^{*}+A^{*} \circ B\right)^{T}=\hat{A}^{T} \circ \hat{B}^{T} .
\end{aligned}
$$

Theorem 3.7 For $\hat{A}, \hat{B} \in \mathbb{D}_{n}^{m}$, if $\hat{A}$ and $\hat{B}$ regular matrices then they satisfy $(\hat{A} \circ \hat{B})^{-1}=$ $\hat{A}^{-1} \circ \hat{B}^{-1}$.

Proof. The inverse of the product of two dual matrices can be shown easily

$$
\begin{aligned}
(\hat{A} \circ \hat{B}) \circ(\hat{A} \circ \hat{B})^{-1} & =\hat{J} \\
\hat{A} \circ\left(\hat{B} \circ(\hat{A} \circ \hat{B})^{-1}\right) & =\hat{J} \\
\hat{B} \circ(\hat{A} \circ \hat{B})^{-1} & =\hat{A}^{-1} \\
(\hat{A} \circ \hat{B})^{-1} & =\hat{A}^{-1} \circ \hat{B}^{-1}
\end{aligned}
$$




\section{Special dual matrices with Hadamard product}

Definition 4.1 Let $\hat{A} \in \mathbb{D}_{n}^{m}$, if all entries of $\hat{A}$ are pure dual numbers then it is called the pure dual matrix.

Example 4.2 $\hat{A}=\left[\begin{array}{cc}2 \varepsilon & 3 \varepsilon \\ -\varepsilon & 7 \varepsilon\end{array}\right]$ is a pure dual matrix.

Definition 4.3 For $\hat{A}, \hat{B} \in \mathbb{D}_{n}^{n}$, Let $\hat{A} \neq 0$ and $\hat{B} \neq 0$ are regular matrices. If $\hat{A} \circ \hat{B}=0$, then $A$ and $B$ are called dual nulling each other matrices.

Definition 4.4 Let $\hat{A} \in \mathbb{D}_{n}^{m}$, the product of $n$ times $\hat{A}$ is called the $n$th power of $\hat{A}$ which is denoted by $\hat{A}^{n}$. Second and third power of matrix $\hat{A}$ can be calculated as follows:

$$
\begin{aligned}
& \hat{A}^{2}=\left[a_{i j} a_{i j}+\varepsilon\left(a_{i j} a_{i j}^{*}+a_{i j}^{*} a_{i j}\right)\right]=\left[a_{i j}^{2}+2 \varepsilon\left(a_{i j} a_{i j}^{*}\right)\right]=A^{2}+2 \varepsilon A A^{*} \\
& \hat{A}^{3}=\left[a_{i j}^{3}+3 \varepsilon a_{i j}^{*} a_{i j}^{2}\right]=A^{3}+3 \varepsilon A^{2} A^{*} .
\end{aligned}
$$

Theorem 4.5 For $\hat{A} \in \mathbb{D}_{n}^{n} n$th power of the matrix $\hat{A}$ is as follows:

$$
\hat{A}^{n}=\left[a_{i j}^{n}+n \varepsilon a_{i j}^{*} a_{i j}^{n-1}\right]=A^{n}+n \varepsilon A^{n-1} A^{*} .
$$

Proof. Desiring result can be obtained easly by mathematical induction.

Definition 4.6 Let $\hat{A} \in \mathbb{D}_{n}^{n}$,

i. $\hat{A}$ is called $\mathbb{D}$ - idempotent matrix which satisfies the property $\hat{A}^{2}=\hat{A}$. There is no $\mathbb{D}-$ idempotent matrix except $\hat{J}$.

ii. If $\hat{A}^{p+1}=\hat{A}$, then it is called $\mathbb{D}$ - periodic dual matrix. Here $p$ is called the period.

iii. If $\hat{A}^{q}=0+\varepsilon 0$, then $\hat{A}$ is called $\mathbb{D}$ - nilpotent dual matrix.

iv. If $\hat{A}^{T}=\hat{A}$, then $\hat{A}$ is called $\mathbb{D}-$ symmetric dual matrix.

v. If $\hat{A}^{T}=-\hat{A}, \hat{A}$ is called $\mathbb{D}$-skew symmetric dual matrix.

vi. If $\hat{A}^{2}=\hat{J}$, then $\hat{A}$ is called $\mathbb{D}-$ involute dual matrix.

vii. If $\left(\hat{A}^{T}\right) \circ \hat{A}=\hat{A} \circ\left(\hat{A}^{T}\right)=\hat{J}_{n}$, then $\hat{A}$ is called $\mathbb{D}$ - orthogonal dual matrix.

\section{Corollary 4.7}

i. There is no $\mathbb{D}$ - idempotent and $\mathbb{D}$ - periodic dual matrix except $\hat{J}$. Moreover, there is just one $\mathbb{D}-$ nilpotent matrix which is zero matrix.

ii. An $n \times n$ matrix $\hat{A}=A+\varepsilon A^{*}$ is $\mathbb{D}$ - (skew)symmetric dual matrix iff $A$ and $A^{*}$ are (skew)symmetric real matrices.

\section{References}

[1] Million, E., “The Hadamard product”, Course Notes 3.6 (2007).

[2] Horn, R. A., Zai Y., "Rank of a Hadamard product", Linear Algebra and its Applications 591 (2020) : 87-98. 
[3] Styan, G. P. H., "Hadamard products and öultivariate statistical analysis", Linear Algebra Appl. 6 (1973) : 217-240.

[4] Horn, R. A., Johnson C. R., “Topics in matrix analysis”, Cambridge University Press, (1994).

[5] Bernstein, D. S., “Matrix mathematics”, Princeton University Press, (2009).

[6] Liu, S., Gotz T., "Hadamard, Khatri-Rao, Kronecker and other matrix products", International Journal of Information and Systems Sciences 4(1) (2008) : 160-177.

[7] Veldkamp G. R., "On the use of dual numbers, vectors and matrices in instantaneous spatial kinematics”, Mechanism and Machine Theory 11 (1976) : 141-156.

[8] Pennestrì, E., Stefanelli R., "Linear algebra and numerical algorithms using dual numbers”, Multibody System Dynamics 18(3) (2007) : 323-344.

[9] Fischer, I. S., "Dual-number methods in kinematics, statics and dynamics", Routledge, (2017).

[10] Dagdeviren, A., “Lorentz matris carpimi ve dual matrislerin ozellikleri”, Master’s Thesis, Yildiz Technical University, (2013). 